\title{
Effect of Adjuvant Drugs in Combination with Propofol on GI Recovery Times: A Retrospective Chart Review
}

Dawood Nasir MD ${ }^{1 *}$, Brandon Mitchell ${ }^{2}$, Syed S. Jeffery, MD ${ }^{3}$, Christi Mary Sunna ${ }^{4}$, Dr. Deepak Aggarwal, MD ${ }^{5}$, Pamela Ereckson Fox MD ${ }^{6}$

\author{
${ }^{1}$ Associate Professor University of Texas, Southwestern Medical Center Dallas, Texas, Dept of Anesthesiology \& Pain Management Ex director Acute \\ Pain Service, Parkland Health \& Hospital Systems \\ ${ }^{2}$ Certified nurse Anesthetist (CRNA) Parkland Health \& Hospital Systems, Dallas, Texas \\ ${ }^{3}$ Resident anesthesia, University of Texas, Southwestern Medical Center Dallas, Texas \\ ${ }^{4}$ Certified Nurse Anesthetist (CRNA) Parkland Health \& Hospital System, Dallas, Texas \\ ${ }^{5}$ Chairman Gastroenterology Dell Seton Medical Center, At the University of Texas, Austin, Texas \\ ${ }^{6}$ Associate Professor University of Texas Southwestern Dept of Anesthesiology \& Pain Management Chairman, Quality Improvement Committee \\ Parkland Health \& Hospital Systems Director, Anesthesia Quality \& Safety Deputy Medical Informatics Officer
}

DOI: $10.36348 /$ sjm.2020.v05i02.014 $\quad$ | Received: 28.12 .2019 | Accepted: 04.01.2020 | Published: 29.02 .2020

*Corresponding author: Dawood Nasir MD

\section{Abstract}

Purpose and objectives: Decrease in length of Post-Anesthesia Care Unit (PACU) recovery time has been linked to both patient satisfaction and reduced healthcare cost. This was a quality project which retrospectively reviewed patients`chart to assess recovery time whether administration of co-analgesics and sedatives in combination with propofol prolong recovery time. Primary outcome: PACU recovery time using the modified Aldrete scoring system. The ideal anesthetic for ambulatory cases should provide rapid and smooth emergence and recovery. Propofol is recently preferred short acting agent for gastrointestinal endoscopic procedures for induction, maintenance of anesthesia/deep sedation (1). The randomized controlled trials indicated that the potential benefits of propofol include a shorter recovery time, greater sedation, and improved patient cooperation (2). However, high dose of propofol may cause side effects and delay emergence. Study Design: This was a quality project which was retrospectively evaluating consecutive Gastroendoscopy cases at Parkland Hospital. Eligible subjects who received esophagogastroduodenoscopy (EGD) in 1 years' period between 2014 to 2015. All patients in the review received propofol as their primary anesthetic, while some received additional co-analgesics and sedatives (midazolam, fentanyl, etomidate, ketamine, diphenhydramine). Recovery and PACU discharge time were compared between the propofol only groups versus the propofol plus adjuvant drug based on the modified Aldrete scoring system. Data was electronically collected in encrypted and password protected Excel file. At the end of the data collection, this Excel file was de-identified for the data analysis. Data was stored in secure encrypted research computer or secure research server and only research team will have access to electronic files. Retrospective analysis of consecutive patients undergoing esophagogastroduodenoscopy (EGD) at Parkland Hospital. The consecutive cases were evaluated for the Postoperative recovery time. The sample size of the project is the expected number of patients underwent EGD during 2014 to 2015.

Keywords: Retrospective data analysis, recovery time, postoperative care, sample size, anesthetic medication, propofol, versed, fentanyl, Aldrete scoring system, discharge criteria.

Copyright @ 2020: This is an open-access article distributed under the terms of the Creative Commons Attribution license which permits unrestricted use, distribution, and reproduction in any medium for non-commercial use (NonCommercial, or CC-BY-NC) provided the original author and source are credited.

\section{BACKGROUND AND AIM}

Decrease in length of Post-Anesthesia Care Unit (PACU) recovery time has been linked to both patient satisfaction and reduced healthcare cost. ${ }^{1-4}$ The aim of this retrospective chart review is to assess whether administration of co-analgesics and sedatives in combination with propofol prolong GI recovery time.

\section{Study Design and Setting}

This was a retrospective chart review of 1,216 who underwent an esophagogastroduodenoscopy (EGD) from November 2018 through April 2019 at a single-center, tertiary referral county hospital. Patients included in this study underwent EGD only, were ASA I, II and III, and between the ages of 18 and 90. Clinical criteria for patient exclusion included patients less than 20 years of age, pregnancy, patients undergoing multiple GI procedures, patients requiring 
endotracheal intubation, and patients undergoing emergency procedures. All patients in the review received propofol as their primary anesthetic, while some received additional co-analgesics and sedatives (midazolam, fentanyl, etomidate, ketamine, diphenhydramine). Recovery and PACU discharge time were compared between the propofol only group versus the propofol plus adjuvant drugs based on the modified Aldrete scoring system.

\section{Outcomes and Measures}

The primary outcome was PACU recovery and discharge time using the modified Aldrete scoring system. Aldrete scoring system is based on patient activity, respiration, circulation, consciousness, color or oxygen saturation, pain, surgical bleeding, nausea and vomiting. The secondary outcome was total procedure duration.

\section{RESULTS}

Of the 1,216 reviewed, 1,022 met our study criteria and were included in our analysis. Of this sample, 591 received propofol only while 431 received propofol and one or more adjuvant drug. A one-tailed independent sample t-test was utilized to compare differences in the two groups. The propofol-only group had quicker recovery and discharge times [(mean time $=56.8$ minutes; $[\mathrm{SD}=41.56]]$ compared to propofol plus adjuvant drug group [(mean time $=60.84$; [SD=49.39])]. However, there was not a statistically significant difference between the two groups $[\mathrm{p}=$ $0.176]$.

\section{CONCLUSION}

Due the relatively short half-life of propofol, it was anticipated that patients receiving propofol as the only anesthetic would demonstrate more rapid recovery and discharge times than those receiving supplemental adjuvants in conjunction with propofol. Compared to the propofol-plus-adjuvant group, our review found no significant difference between PACU recovery and discharge times for patients undergoing EGDs who received propofol as their only anesthetic. Other identifiable causes for delays irrespective of group included patient transportation issues, delayed or no bed availability, patient co-morbidities requiring consult services, and improper or missing physician orders needed for discharge.

\section{REFERENCES}

1. Delgado, A.A.A., de, Mauro, D.T.H., Ribiero, I.B., Bazarbashi, A.N., Dos, Santos, M.E.L., Bernando, W.N., de, Mauro, M.G.H. (2019). Propofol versus traditional sedation in endoscopy: A systematic review and meta-analysis. World Journal of Gastrointestinal Endoscopy, 11(12): 573-588.

2. Helmers, R. A., Dilling, J. A., Chaffee, C. R., Larson, M. V., Narr, B. J., Haas, D. A., \& Kaplan, R. S. (2017). Overall cost comparison of gastrointestinal endoscopic procedures with endoscopist-or anesthesia-supported sedation by activity-based costing techniques. Mayo Clinic Proceedings: Innovations, Quality \& Outcomes, 1(3), 234-241.

3. Padmanabhan, U., Leslie, K., Eer, A. S., Maruff, P., \& Silbert, B. S. (2009). Early cognitive impairment after sedation for colonoscopy: the effect of adding midazolam and/or fentanyl to propofol. Anesthesia \& Analgesia, 109(5), 14481455.

4. Qadeer, M. A., Vargo, J. J., Khandwala, F., Lopez, R., \& Zuccaro, G. (2005). Propofol versus traditional sedative agents for gastrointestinal endoscopy: a meta-analysis. Clinical Gastroenterology and Hepatology,3(11), 10491056.

5. Eberl, S., Koers, L., van Hooft, J. E., de Jong, E., Schneider, T., Hollmann, M. W., \& Preckel, B. (2017). Sedation with propofol during ERCP: is the combination with esketamine more effective and safer than with alfentanil? Study protocol for a randomized controlled trial. Trials, 18(1), 472.

6. Dos Santos, M. E. L., Maluf-Filho, F., Chaves, D. M., Matuguma, S. E., Ide, E., de Oliveira Luz, G., .. \& Sakai, P. (2013). Deep sedation during gastrointestinal endoscopy: propofol-fentanyl and midazolam-fentanyl regimens. World Journal of Gastroenterology: WJG, 19(22), 3439. 